\title{
The Potential Effect of Metformin on Cancer: An Umbrella Review
}

\author{
Hong Yu ${ }^{\dagger}, X_{i}$ Zhong $^{\dagger}$, Peng Gao, Jinxin Shi, Zhonghua Wu, Zhexu Guo, Zhenning Wang * \\ and Yongxi Song*
}

Department of Surgical Oncology and General Surgery, The First Hospital of China Medical University, Shenyang, China

\section{OPEN ACCESS}

Edited by:

Roberta Malaguarnera Università degli studi Magna Græcia di

Catanzaro, Italy

Reviewed by:

Jean-Daniel Lalau,

University Hospital Center (CHU) of

Amiens, France

Ernestina Marianna De Francesco,

University of Manchester,

United Kingdom

Rosario Le Moli,

University of Catania, Italy

*Correspondence:

Zhenning Wang

josieon826@sina.cn

Yongxi Song

songyongxi840309@126.com

tThese authors have contributed equally to this work

Specialty section:

This article was submitted to Cancer Endocrinology, a section of the journal

Frontiers in Endocrinology

Received: 15 May 2019

Accepted: 27 August 2019

Published: 18 September 2019

Citation:

Yu H, Zhong X, Gao P, Shi J, Wu Z, Guo Z, Wang Z and Song Y (2019) The Potential Effect of Metformin on

Cancer: An Umbrella Review.

Front. Endocrinol. 10:617.

doi: 10.3389/fendo.2019.00617
Background: Metformin has been reported to possess anti-cancer properties in addition to glucose-lowering activity and numerous systematic reviews and meta-analyses have studied the association between metformin use and cancer incidence or survival outcomes. We performed an umbrella review to assess the robustness of these associations to facilitate proper interpretation of these results to inform clinical and policy decisions.

Methods: We searched PubMed and Embase systematic reviews and meta-analyses investigating the effect of metformin use on cancer incidence or survival outcomes published from inception to September 2, 2018. We estimated the summary effect size, the $95 \% \mathrm{Cl}$, and the $95 \%$ prediction interval, heterogeneity, evidence of small-study effects, and evidence of excess significance bias.

Results: We included 21 systematic reviews and meta-analyses covering 11 major anatomical sites and 33 associations. There was strong evidence for the association between metformin use and decreased pancreatic cancer incidence. The association between metformin use and improved colorectal cancer overall survival (OS) was supported by highly suggestive evidence. Seven associations (all cancer incidence, all cancer OS, breast cancer OS, colorectal cancer incidence, liver cancer incidence, lung cancer OS, and pancreatic cancer OS) presented only suggestive evidence. The remaining 24 associations were supported by weak or not-suggestive evidence.

Conclusions: Associations between metformin use and pancreatic cancer incidence or colorectal cancer OS are supported by strong or highly suggestive evidence, respectively. However, these results should be interpreted with caution due to the poor methodological quality of the systematic reviews and meta-analyses.

Keywords: metformin, cancer, umbrella review, drug re-purposing, decision-making

\section{INTRODUCTION}

Cancer is one of the largest problems in the world at present, ranking second among all factors causing death in the United States every year (1). Current treatment methods for cancer include surgical resection, radiotherapy, and chemotherapy. Due to rapid tumor cell growth, easy metastasis, chemoradiotherapy resistance, and other biological characteristics, it is difficult for conventional treatments to completely remove tumor cells, contributing to poor prognosis. Drug re-purposing has been used to ensure the safety of drugs and avoid long cycles of drug development and screening. Today, some well-established drugs such as aspirin (2) and digoxin (3) have been found to have anti-tumor effects and have been applied in new fields. 
Metformin is a semi-synthetic oral hypoglycemic agent which mainly reduces blood glucose by activating the adenosine monophosphate activated protein kinase (AMPK) signaling pathway, inhibiting hepatic glucose output, improving peripheral tissue sensitivity to glucose, and increasing glucose uptake (4). The definite curative effect, good safety, and low cost have allowed metformin to be recommended as the first-line oral treatment for type 2 diabetes mellitus (T2DM) and the most commonly prescribed drug in T2DM patients. In 1998, British scientists found that metformin had a protective effect on the cardiovascular system (5), inspiring researchers to work on re-purposing metformin. Subsequent studies reported that metformin can be used for adjuvant treatment of tuberculosis (6) and for routine treatment of polycystic ovary syndrome (7).

In recent years, epidemiological data have shown that diabetes increases the risk of breast cancer, colorectal cancer, pancreatic cancer, endometrial cancer, and other malignant tumors. In 2005, Scottish researchers found that diabetic patients taking metformin had a lower risk of cancer, indicating that metformin may possess anti-tumor abilities (8). According to another study, metformin likely has an inhibitory effect on tumor progression in patients with $\mathrm{T} 2 \mathrm{DM}$, which can reduce the risk of tumor and tumor-related mortality of patients, improving their survival rate (9). An increasing number of observational studies and randomized controlled trials (RCT) have studied the association between metformin use and cancer incidence or survival outcomes (10), and a mass of systematic reviews and meta-analyses have been conducted to evaluate the relationship between metformin and cancer (11). The observed associations between may exaggerate the effects of metformin on cancer, as substantial heterogeneity and potential biases reside in the included systematic reviews. We performed an umbrella review to provide a comprehensive review of claimed associations between metformin use and cancer risk or survival outcomes for different cancers and critically assess the robustness of these associations to facilitate proper interpretation of these results to inform clinical and policy decisions.

\section{METHODS}

\section{Protocol and Study Design}

The protocol for conducting this umbrella review of systematic reviews and meta-analyses exploring the effect of metformin use on cancer risk or survival outcomes was developed accordingly:

\section{Search Strategy and Eligibility Criteria}

Two researchers (HY and XZ) independently searched PubMed and Embase systematic reviews and meta-analyses investigating the effect of metformin use on cancer incidence or survival outcomes published from inception to September 2, 2018. The search strategy used the following terms: metformin AND (cancer OR tumor OR neoplasm OR malignan*) AND (systematic OR meta-analysis). Two authors (HY, XZ) independently reviewed the titles and abstracts, carefully read the full text of potential eligible studies, and completed the study selection.
Included eligible systematic reviews and meta-analyses addressed associations between metformin use and cancer incidence and/or survival outcomes, including overall survival (OS), recurrence-free survival (RFS), cancer-specific survival (CSS), and progression-free survival (PFS). Articles were also included if studies assessed different groups of people with the same cancer or focused on subtypes of a particular cancer. When two or more meta-analyses were found on the same association, only the one containing the most primary studies was included to avoid duplication.

\section{Data Extraction}

Two investigators (HY, XZ) independently extracted data from eligible articles, retrieving first author name; publication year; cancer type; number of included studies; number of cases and population size; relative risk estimates including risk ratio (RR), odds ratio (OR), and hazard ratio (HR); and the corresponding 95\% confidence interval (CI) from the eligible systematic reviews and meta-analyses. Divergences were resolved through discussion. First author, number of cases and population size, relative risk estimates ( $\mathrm{RR}, \mathrm{OR}$, and $\mathrm{HR}$ ), and corresponding 95\% CI were extracted from each individual study in included systematic reviews or meta-analyses for further analysis.

\section{Quality Assessment}

Each included systematic review and meta-analysis was independently assessed by two authors (HY, XZ) using the Assessing the Methodological Quality of Systematic Reviews version 2.0 (AMSTAR 2.0) tool (12). AMSTAR 2.0 measures 16 items, provides a comprehensive rationale for item selection, identifies critical domains, and rates the validity of the results of the review as high, moderate, low, or critically low instead of creating an overall score. These features make AMSTAR 2.0 a major upgrade to AMSTAR. Discrepancies were resolved by consensus.

\section{Statistical Analysis}

\section{Assessment of Summary Effect and Heterogeneity}

For each meta-analysis on the association between metformin use and cancer risk or survival outcomes, the summary effect was synthesized and its 95\% CI was calculated using random-effect models. Inter-study heterogeneity was evaluated with Cochran's Q-test and the $I^{2}$ statistic (13). Statistical inconsistencies could demonstrate either genuine inter-study heterogeneity or underlying bias. The $95 \% \mathrm{CI}$ of $I^{2}$ was calculated to assess the uncertainty around heterogeneity estimates (14).

\section{Estimation of Prediction Intervals}

Ninety five percentage prediction intervals (PI) for the summary random effect estimates were calculated to further interpret interstudy heterogeneity and represent the prediction of the effect in an individual-study setting (15).

\section{Assessment of Small-Study Effects}

Small study effects can indicate publication bias, genuine heterogeneity, or chance. The Egger's regression asymmetry test was used to detect small study effect biases. Small study effect bias was considered to exist when the Egger's test $P<0.10$ (16). 


\section{Evidence of Excess Significance Bias}

The excess significance test evaluated whether the actual observed number $(\mathrm{O})$ of positive studies $(P<0.05)$ was different from the expected number of studies (E) with statistically significant results (17). E was the sum of the statistical power estimates for each component study in each meta-analysis and was calculated with an algorithm using a non-central $t$ distribution. In cases in which $O>E$ AND $P<0.10$, the excess significance test was considered positive.

\section{$10 \%$ Credibility Ceiling}

Credibility ceiling analyses were performed to account for the innate methodological limitations of observational studies (18). The level was set at $10 \%$ to re-estimate the inter-study heterogeneity and summary relative risk between studies.

\section{Grading the Existing Evidence}

Nominally statistically significant $(P<0.05)$ associations from meta-analyses exploring the effect of metformin use on cancer risk or survival outcomes were classified into four levels-strong $\left[P<10^{-6},>1,000\right.$ cases, $P<0.05$ of the largest component study in the meta-analysis, no large heterogeneity $\left(I^{2}<50 \%\right)$, no evidence of small-study effects $(P>0.1$ for Egger's test), the 95\% PI excludes the null value (1), no excess significance bias $(P>0.1)$, and survives the $10 \%$ credibility ceiling test $(P>0.05)]$, highly suggestive $\left(P<10^{-6},>1,000\right.$ cases, $P<0.05$ of the largest component study in the meta-analysis), suggestive $\left(P<10^{-3}\right.$, $>1,000$ cases $)$, and weak $(P<0.05)(19,20)$.

All statistical analyses were performed using STATA version 12.0 .

\section{RESULTS}

\section{Characteristics of the Included Systematic Reviews and Meta-Analyses}

Of the 808 records obtained from the literature search through PubMed and Embase, we ultimately included 21 systematic reviews covering 33 associations (11, 21-40). The search flowchart and reasons for excluding 787 are shown in Figure 1. The 21 studies included 11 major anatomical sites (bladder, breast, colorectum, gastric, endometrium, ovarian, kidney, liver, lung, pancreas and prostate), 33 different associations between metformin use and cancer risk or survival outcomes, 327 primary studies, more than 206,000 cases and more than 13 million subjects. Of note, all subjects were diagnosed with T2DM at baseline. The characteristics of the 33 associations are shown in Figures 2, 3, and the full database of the 327 primary studies is available in Supplementary Table 4.

\section{Quality Assessment Using AMSTAR 2.0}

We used the 16-item AMSTAR 2.0 to assess the methodological quality of the 21 eligible systematic reviews, and all qualities were considered critically low. All included studies had more than one critical flaw [usually in items 2 (18/21, 85.7\%), 7 (21/21, 100\%), and $13(21 / 21,100 \%)$ ] and several non-critical flaws [usually in items 3 (18/21, 85.7\%), 10 (21/21, 100\%), and 12 (19/21, 90.5\%)]. It should be noted that reviews with more than one critical flaw, regardless of non-critical weaknesses, should be interpreted with caution for a credible summary of the existing evidence. The detailed results, scoring criteria, and rating criteria are shown in Supplementary Table 1.

\section{Summary Effect Size}

Twenty three of the 33 associations in the included meta-analyses were statistically significant with a threshold of $P<0.05$, with the remaining 10 associations presenting $P>0.05$. Of the statistically significant associations, five reached $P<10^{-6}$ : associations between metformin use and pancreatic cancer incidence, colorectal cancer OS, all cancer incidence, breast cancer OS, or liver cancer incidence (Tables 1, 2; Supplementary Tables 2, 3). Associations between metformin use and all cancer OS, colorectal cancer incidence, lung cancer OS, pancreatic cancer OS, all cancer CSS, or endometrial cancer OS reached a moderate statistical significance $\left(P<10^{-3}\right)$. The remaining 12 associations including bladder cancer CSS, bladder cancer RFS, advanced colorectal adenoma incidence, colorectal cancer CSS, gastric cancer incidence, endometrial cancer RFS, ovarian cancer incidence, kidney cancer OS, kidney cancer CSS, liver cancer OS, prostate cancer OS and prostate cancer RFS reached $P$ $<0.05$. The combined results demonstrate that metformin can decrease cancer risk or increase survival outcome among all associations with strongly statistically significant summary random effect estimates.

\section{Heterogeneity}

There were 10 associations with moderate to high heterogeneity $\left(I^{2}=50-75 \%\right)$ and 12 associations with high heterogeneity $\left(I^{2}\right.$ $>75 \%)$. When we calculated the 95\% PI to further assess interstudy heterogeneity, we found only one association with the null value excluded (pancreatic cancer incidence) (Tables 1, 2; Supplementary Tables 2, 3).

\section{Small-Study Effects}

Of the 33 associations between metformin use and cancer incidence or survival outcomes, small study effects were detected in nine (colorectal cancer OS, all cancer incidence, all cancer OS, breast cancer OS, liver cancer incidence, gastric cancer incidence, colorectal adenoma incidence, bladder cancer incidence and prostate cancer incidence) according to the Egger's test $(P<0.1)$ as shown in Tables 1, 2; Supplementary Tables 2, 3.

\section{Excess Significance}

Nine associations (all cancer incidence, all cancer OS, breast cancer OS, liver cancer incidence, bladder cancer CSS, bladder cancer RFS, gastric cancer incidence, liver cancer OS, and bladder cancer OS) had evidence of excess significance bias $(P<0.1$ AND $O>E$ ) (Tables 1, 2; Supplementary Tables 2, 3).

\section{$10 \%$ Credibility Ceiling}

Twelve associations (pancreatic cancer incidence, colorectal cancer OS, all cancer OS, breast cancer OS, liver cancer incidence, lung cancer OS, pancreatic cancer OS, all cancer CSS, endometrial cancer RFS, kidney cancer OS, kidney cancer CSS, and liver cancer OS) remained statistically significant with credibility ceilings set at $10 \%$. 


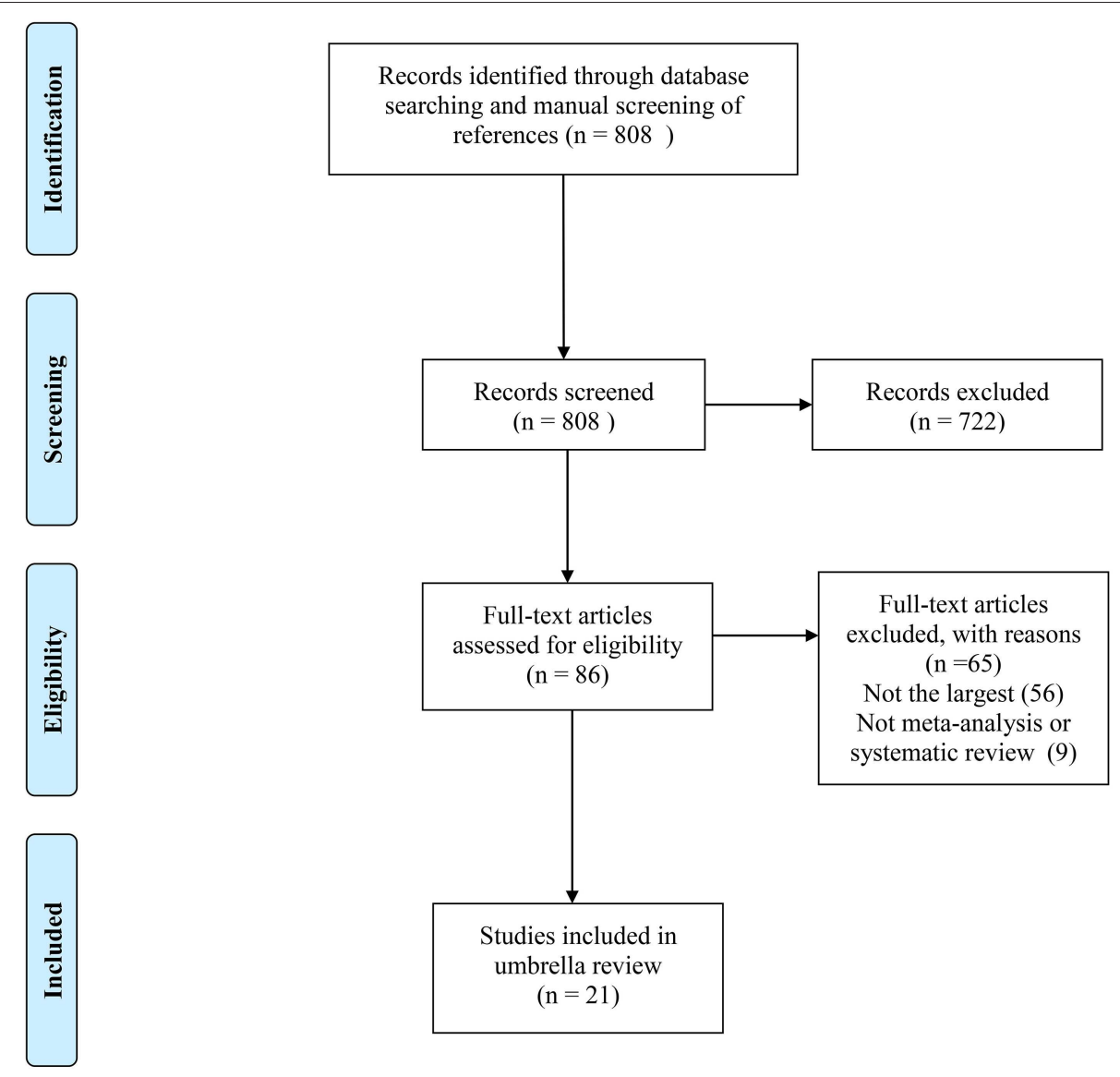

FIGURE 1 | The flow diagram of study selection.

\section{Robustness of Evidence}

We found that 24 of 33 associations between metformin use and cancer risk or survival outcomes were supported by weak or notsuggestive evidence (Tables 1, 2; Supplementary Tables 2, 3). There was strong evidence for the association between metformin use and pancreatic cancer incidence. The association between metformin use and colorectal cancer OS was supported by highly suggestive evidence. The remaining 7 associations (all cancer incidence, all cancer OS, breast cancer OS, colorectal cancer incidence, liver cancer incidence, lung cancer OS, and pancreatic cancer OS) presented only suggestive evidence.

\section{DISCUSSION}

\section{Main Findings and Interpretation in Light of Existing Evidence}

In this umbrella review of systematic reviews and metaanalyses evaluating the current evidence for associations between metformin use and cancer risk or survival outcomes, we summarized 21 studies covering 11 major anatomical sites, 327 primary studies, more than 206,000 cases, and over 13 million subjects. According to statistical data analyses, the association between metformin and pancreatic cancer incidence was supported by strong evidence, suggesting that metformin may be associated with decreased risk of pancreatic cancer. We additionally found that the association between metformin and colorectal cancer OS had highly suggestive evidence, indicating that patients with colorectal cancer using metformin likely have a better OS than those not using metformin.

Pancreatic cancer ranks fourth for cancer-related deaths worldwide (41). However, just $10-20 \%$ of the patients are eligible for surgical treatment because of the late diagnosis (42). Several studies showed significantly reduced risk of pancreatic cancer in T2DM patients treated with metformin compared with those without $(43,44)$. It is reported that metformin inhibits proliferation of pancreatic cancer cells through the AMPK/mammalian target of rapamycin (mTOR) axis and by down-regulating activity of the insulin/insulin-like growth factor signaling pathway $(45,46)$. In our study, evidence for associations between metformin and pancreatic cancer was strong for incidence and suggestive for OS. Therefore, policy makers should cautiously consider metformin for routine use to protect against pancreatic cancer or as an option in treating pancreatic cancer patients, especially those with T2DM.

Colorectal cancer ranks as the third most common malignant tumor in the world. About 600 thousand people die of colorectal 


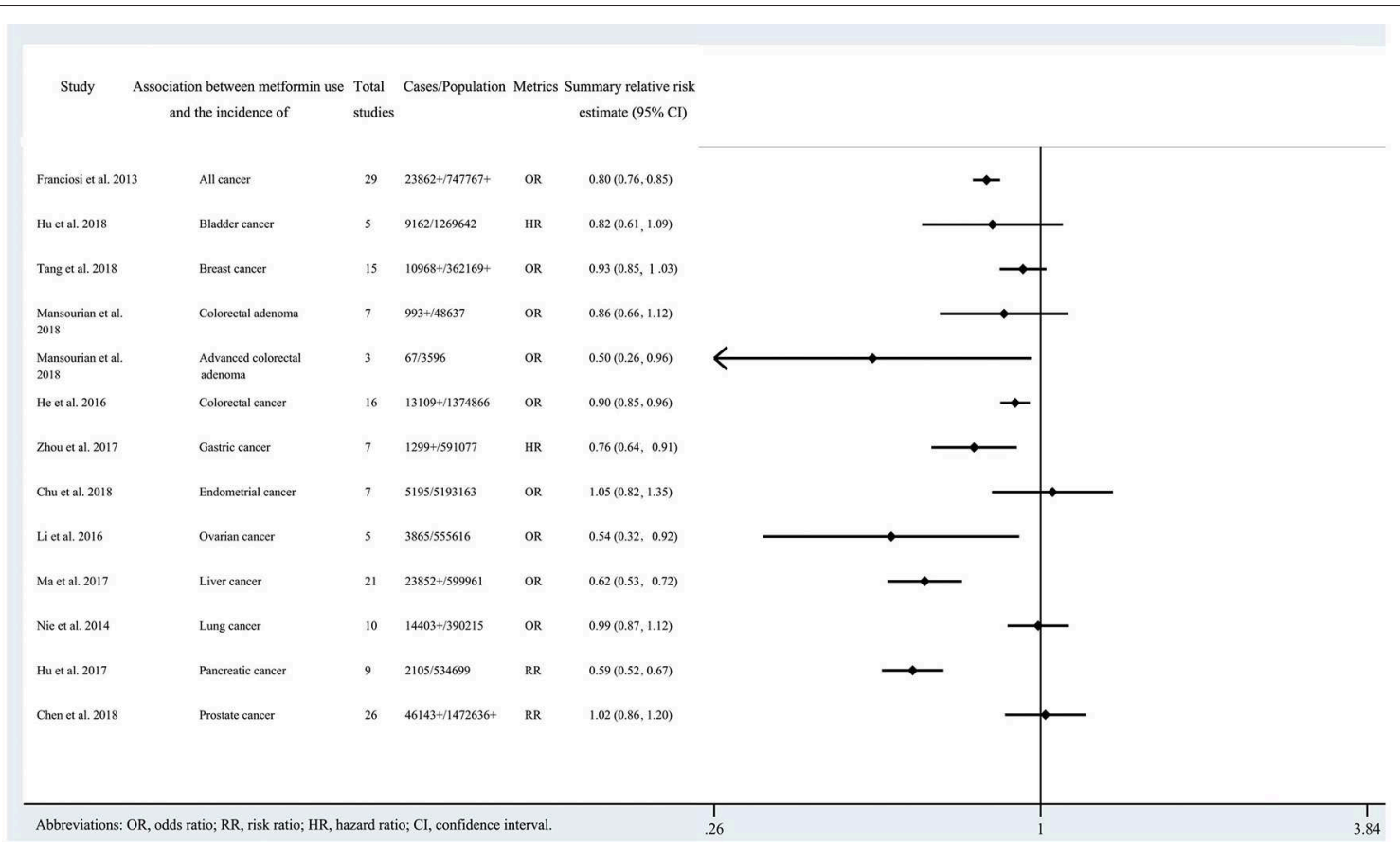

FIGURE 2 | Characteristics of the 13 associations on cancer incidence in the included systematic reviews and meta-analyses.

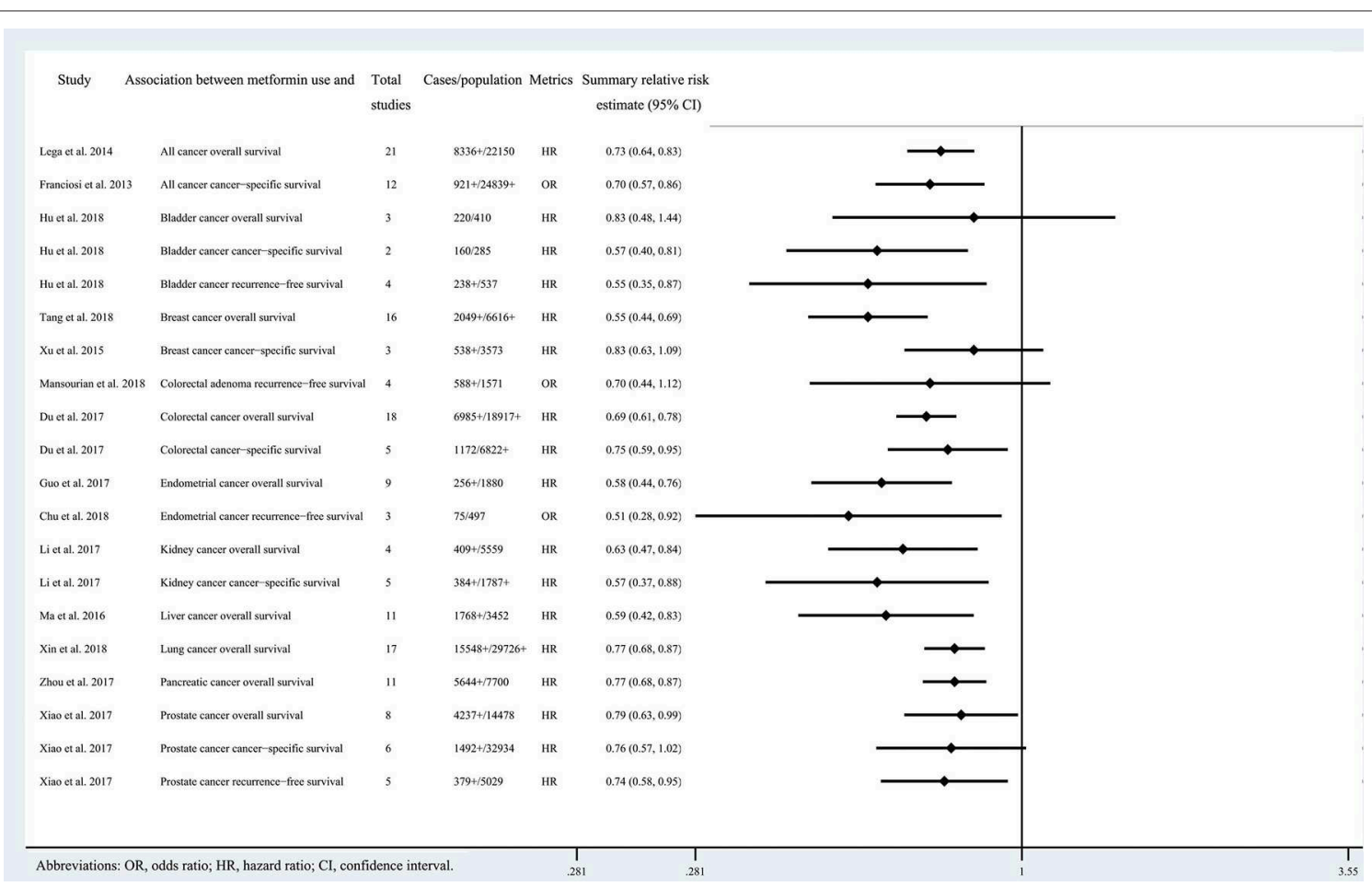

FIGURE 3 | Characteristics of the 20 associations on cancer prognosis in the included systematic reviews and meta-analyses.

cancer every year worldwide (47). It has been reported that metformin intake is associated with reduced risk of colorectal cancer and improved survival in colorectal cancer patients
$(48,49)$. These results are consistent with the findings in our study as we found highly suggestive evidence for colorectal cancer OS and suggestive evidence for colorectal cancer risk. 
TABLE 1 | Evidence-rating results based on the results of statistical analyses of the 13 associations on cancer incidence.

\begin{tabular}{|c|c|c|c|c|c|c|c|c|c|}
\hline Study & $\begin{array}{l}\text { Association between } \\
\text { metformin use and the } \\
\text { incidence of }\end{array}$ & $\begin{array}{c}\text { Summary relative } \\
\text { risk estimate } \\
(\text { random-effect } P)^{*}\end{array}$ & $\begin{array}{c}\text { Cases } \\
>1,000\end{array}$ & $\begin{array}{l}\text { Largest study } \\
\text { relative risk estimate } \\
P<0.05\end{array}$ & $\begin{aligned} & I^{2} \\
< & 50 \%\end{aligned}$ & $\begin{array}{l}\text { Small } \\
\text { study } \\
\text { effects }\end{array}$ & $\begin{array}{c}95 \% \text { prediction } \\
\text { interval exclude } \\
\text { the null value }\end{array}$ & $\begin{array}{c}\text { Excess } \\
\text { significance }\end{array}$ & $\begin{array}{c}\text { Ten percentage } \\
\text { credibility } \\
\text { ceiling survival }\end{array}$ \\
\hline \multicolumn{10}{|c|}{ Associations supported by strong evidence (1) } \\
\hline Hu et al. (38) & Pancreatic cancer & +++ & + & + & + & - & + & - & + \\
\hline \multicolumn{10}{|c|}{ Associations supported by suggestive evidence (3) } \\
\hline Franciosi et al. (21) & All cancer & +++ & + & - & - & + & - & + & - \\
\hline He et al. (27) & Colorectal cancer & ++ & + & + & + & - & - & - & - \\
\hline Ma et al. (34) & Liver cancer & +++ & + & - & - & + & - & + & + \\
\hline \multicolumn{10}{|c|}{ Associations supported by weak evidence (3) } \\
\hline Mansourian et al. (26) & Advanced colorectal adenoma & + & - & + & - & - & - & - & - \\
\hline Zhou et al. (29) & Gastric cancer & + & + & + & - & + & - & + & - \\
\hline Li et al. (32) & Ovarian cancer & + & + & + & - & - & - & - & - \\
\hline \multicolumn{10}{|c|}{ Associations supported by not suggestive evidence (6) } \\
\hline Hu et al. (23) & Bladder cancer & - & + & + & - & + & - & - & - \\
\hline Tang et al. (24) & Breast cancer & - & + & + & + & - & - & - & - \\
\hline Mansourian et al. (26) & Colorectal adenoma & - & - & + & - & + & - & - & - \\
\hline Chu et al. (30) & Endometrial cancer & - & + & + & - & - & - & - & - \\
\hline Nie et al. (36) & Lung cancer & - & + & + & - & - & - & - & - \\
\hline Chen et al. (11) & Prostate cancer & - & + & + & - & + & - & - & - \\
\hline
\end{tabular}

${ }^{*} P$-value calculated using random-effect model:,$+++ P<10^{-6} ;++, P<10^{-3} ;+, P<0.05 ;-, P>0.05$. For other items, $+=$ yes, $-=n o$. 
TABLE 2 | Evidence-rating results based on the results of statistical analyses of the 20 associations on cancer prognosis.

\begin{tabular}{|c|c|c|c|c|c|c|c|c|c|}
\hline Study & $\begin{array}{l}\text { Association between } \\
\text { metformin use and }\end{array}$ & $\begin{array}{l}\text { Summary relative } \\
\text { risk estimate } \\
\text { (random-effect } P)^{*}\end{array}$ & $\begin{array}{l}\text { Cases } \\
>1000\end{array}$ & $\begin{array}{l}\text { Largest study } \\
\text { relative risk estimate } \\
P<0.05\end{array}$ & $\begin{array}{c}I^{2} \\
<50 \%\end{array}$ & $\begin{array}{l}\text { Small } \\
\text { study } \\
\text { effects }\end{array}$ & $\begin{array}{l}95 \% \text { prediction } \\
\text { interval exclude } \\
\text { the null value }\end{array}$ & $\begin{array}{c}\text { Excess } \\
\text { significance }\end{array}$ & $\begin{array}{l}10 \% \text { credibility } \\
\text { ceiling survival }\end{array}$ \\
\hline
\end{tabular}

Associations supported by highly suggestive evidence (1)

Associations supported by suggestive evidence (4)

Lega et al. (22) All cancer overall survival

Tang et al. (24) Breast cancer overall survival

Xin et al. (37) Lung cancer overall survival

Zhou et al. (29) Pancreatic cancer overall survival

Associations supported by weak evidence (11)

Franciosi et al. (21) All cancer cancer-specific

Hu et al. (23) Bladder cancer cancer-specific

survival

Hu et al. (23) Bladder cancer recurrence-free

survival

Du et al. (28)

Colorectal cancer

cancer-specific survival

Guo et al. (31)

Endometrial cancer overall

survival

Chu et al. (30)

Endometrial cancer

Li et al. (33)

recurrence-free survival

Li et al. (33)

Kidney cancer overall survival

Ma et al. (35)

Kidney cancer cancer-specific

survival

Xiao et al. (40)

Liver cancer overall survival

Xiao et al. (40)

Prostate cancer overall survival

Prostate cancer recurrence-free survival

Associations supported by not suggestive evidence (4)

Hu et al. (23) Bladder cancer overall survival

Xu et al. (25) Breast cancer cancer-specific

survival

Mansourian et al. (26) Colorectal adenoma

Xiao et al. (40)

recurrence-free survival

Prostate cancer cancer-specific

survival

${ }^{*} P$-value calculated using random-effect model:,$+++ P<10^{-6} ;++, P<10^{-3} ;+, P<0.05 ;-, P>0.05$. For other items, $+=$ yes, $-=$ no.

\#Hu et al. (23) included only two primary studies, thus Egger's test and prediction interval are not available. 
Our findings may shed light on the treatment or prevention of colorectal cancer with metformin.

With the development of medical science and the continuous discussion and practice of drugs, drug repurposing refers to the drugs that have been put on the market for a long time and have been known by the majority of medical groups, seek new medical treatments from among existing medications rather than through the development of de novo medicines (50). Drug repurposing is based on previous research and development. Detailed formulation, mechanism and safety information of drugs are known, which means that compared with brandnew drugs, old drugs with new uses have obvious advantages of low research cost, low risk and high success rate, and can be put into clinical trials more quickly. Drug repurposing is a kind of innovation in the deep exploration of pharmacological mechanism in clinical practice. As a drug development strategy, it has received more and more attention, and a large number of new drugs for indications have been born. For example, dapoxetine for premature ejaculation is based on drug side effects; thalidomide for the treatment of multiple myeloma is based on the existing mechanism.

Metformin is a safe and effective biguanide hypoglycemic agent, which mainly activates AMPK signaling pathway, decreases hepatic glucose output, promotes the uptake of glucose in peripheral tissues, increases insulin sensitivity, inhibits intestinal cells from absorbing glucose, promotes the secretion of GLP-1 (51) and affect gut microbiota (52). Recent studies have found that metformin has the effects of weight loss, anti-aging and anti-cardiovascular disease. With the increasing awareness of metformin on cancer, numerous studies have shown that metformin can be used to reduce the incidence of cancer and improve the prognosis of cancer patients. Diabetes patients taking metformin reduced their risk of cancer by 30 to 50 percent, especially the risk of pancreatic, hepatocellular, and colon cancers (53).

There have been substantial reports on the mechanism of metformin's anti-tumor effect. Metformin activates AMPK and induces G1 phase arrest of the cell cycle by inhibiting cyclin D1 expression (54). Activation of AMPK can increase the expression of p53 gene and play an anti-tumor role. Low concentration of metformin can induce p53-dependent cell senescence of liver cancer cells by activating AMPK (55). Metformin can also inhibit tumor growth through the mTOR signaling in AMPK-dependent and -independent pathway (56). Treatment with metformin lowers serum levels of insulin and insulin-like growth factor1 (IGF-1), which are both potential growth factors capable of stimulating cell survival and mitogenesis $(57,58)$. Furthermore, a recent study has reported the effect of metformin on androgeninduced IGF-I receptor (IGF-IR) upregulation resulting in the reduction of IGF1-mediated biological effects in prostate cancers cells (59). Normal cells generate adenosine-triphosphate (ATP) mainly through mitochondrial oxidative phosphorylation, while most tumor cells prefer anaerobic glycolysis as an energy generation approach, which is the Warburg effect (60). AMPK activation inhibits fatty acid synthetase and acetyl-CoA carboxylase, leading to reduced fatty acid production in tumor cells, which inhibits tumor cells proliferation. In the study of the tricarboxylic acid cycle, molecular oxygen is reduced to deuterium depleted water, which affects gluconeogenesis as well as fatty acid oxidation, by the terminal complex of mitochondrial electron transport chain (61). Deuterium depleted water is proposed to delay tumor progression using natural ketogenic diets and low deuterium drinking water (62). This action is shared by other biguanides (such as phenformin), statins, and gleevec based on their structural similarities (63). These findings may open up new oncology directions toward metabolically driven submolecular targets to prevent and treat cancers.

Previous studies have shown that high glucose can provide the optimal growth environment for tumors. On the other hand, the hypoglycemic effect of metformin may mask the regulatory effect of metformin on related pathways in tumor therapy. Therefore, it is worth considering whether metformin is effective in patients with pre-diabetes, normal blood glucose or even hypoglycemia. Elgendy et al. (64), found that intermittent fasting in combination with metformin can inhibit the glycolysis and oxidative phosphorylation of tumor cells, namely the drug showing the best antitumor effect with low blood glucose level. At the meanwhile, the strongest anti-tumor effect of metformin at hypoglycemia is independent of AMPK activity. Zhuang et al. (65) also found that low glucose can enhance the toxicity of metformin in breast and ovarian cancer cells and reduce the intracellular ATP level. However, considering the literature body of current evidence, the application of metformin to patients with normal blood glucose remains to be discussed. The effects of metformin on patients' blood glucose and the side effects of metformin are all worthy of clinicians' caution.

\section{Strengths and Limitation}

We performed this detailed umbrella review to evaluate the evidence of associations between metformin and cancer risk or survival outcomes and applied statistical analyses to assess robustness and validity. In addition, we used a comprehensive and systematic criterion to grade evidence levels to rate the strength of these systematic reviews and meta-analyses.

Our review inevitably has limitations and drawbacks. First, we fully trust the accuracy of the data provided in the included meta-analyses. As such, problems within the published data may impact the evidence-rating results despite our statistical analyses. Second, meta-analyses that include $<10$ studies did not allow for statistical tests to identify small study effects and excess significance. Third, the methodological quality of all included systematic reviews and meta-analyses were considered critically low, and future studies that meet the stringent criteria of AMSTAR 2.0 should be conducted to further confirm the findings in our study.

\section{CONCLUSION}

In conclusion, associations between metformin use and pancreatic cancer incidence or colorectal cancer OS are supported by strong or highly suggestive evidence, respectively. However, these results should be interpreted with caution and no firm conclusion can be drawn due to the poor methodological quality of the systematic reviews and meta-analyses. 


\section{DATA AVAILABILITY}

All datasets generated for this study are included in the Supplementary Table 4.

\section{AUTHOR CONTRIBUTIONS}

YS, ZWa, and XZ conceived and designed the study. HY and $\mathrm{XZ}$ performed the literature search, acquired, and collated the data, which were analyzed by XZ, PG, JS, ZWu, and ZG. All authors drafted, critically revised the manuscript for important intellectual content, and gave final approval of the version to be published and contributed to the manuscript. YS and ZWa are guarantors. YS and ZWa attest that all listed authors meet authorship criteria and that no others meeting the criteria have been omitted.

\section{FUNDING}

This work was supported by National Key R\&D Program of China (MOST-2017YFC0908300, MOST-2017YFC0908305),

\section{REFERENCES}

1. Siegel RL, Miller KD, Jemal A. Cancer statistics, 2016. CA Cancer J Clin. (2016) 66:7-30. doi: 10.3322/caac. 21332

2. Chan A. The role of aspirin in colorectal cancer prevention and treatment. Oncology. (2013) 27:1012, 1014, 1042.

3. Flahavan EM, Sharp L, Bennett K, Barron TI. A cohort study of digoxin exposure and mortality in men with prostate cancer. BJU Int. (2014) 113:23645. doi: 10.1111/bju. 12287

4. Luengo A, Sullivan LB, Heiden MGV. Understanding the complex-I-ty of metformin action: limiting mitochondrial respiration to improve cancer therapy. BMC Biol. (2014) 12:82. doi: 10.1186/s12915-014-0082-4

5. Turner RC, Holman RR, Stratton IM, Cull CA, Matthews DR, Manley SE. Effect of intensive blood-glucose control with metformin on complications in overweight patients with type 2 diabetes (UKPDS 34). UK Prospective Diabetes Study (UKPDS) Group. Lancet. (1998) 352:854-65. doi: 10.1016/S0140-6736(98)07037-8

6. Kaswala DH. Drug rash with eosinophilia and systemic symptoms syndrome due to anti-TB medication. I Fam Med Primary Care. (2013) 2:83-5. doi: 10.4103/2249-4863.109958

7. Graff SK, Mario FM, Ziegelmann P, Spritzer PM. Effects of orlistat vs. metformin on weight loss-related clinical variables in women with PCOS: systematic review and meta-analysis. Int J Clin Pract. (2016) 70:450-61. doi: $10.1111 /$ ijcp.12787

8. Clarke PM, Gray AM, Briggs A, Stevens RJ, Matthews DR, Holman RR. Cost-utility analyses of intensive blood glucose and tight blood pressure control in type 2 diabetes (UKPDS 72). Diabetologia. (2005) 48:868-77. doi: 10.1007/s00125-005-1717-3

9. Libby G, Donnelly LA, Donnan PT, Alessi DR, Morris AD, Evans JM. New users of metformin are at low risk of incident cancer: a cohort study among people with type 2 diabetes. Diabetes Care. (2009) 32:1620-5. doi: $10.2337 / \mathrm{dc} 08-2175$

10. Bansal M, Siegel E, Govindarajan R. The effect of metformin (M) on overall survival (OS) of patients (Pts) with colorectal cancer (CRC) treated with chemotherapy (CTX). J Clin Oncol. (2011) 29:2696. doi: 10.1200/jco.2011.29.15_suppl.2608

11. Chen CB, Eskin M, Eurich DT, Majumdar SR, Johnson JA. Metformin, Asian ethnicity and risk of prostate cancer in type 2 diabetes: a systematic review and meta-analysis. BMC Cancer. (2018) 18:65. doi: 10.1186/s12885-017-3934-9
Program for Liaoning Innovative Research Team in University (LT2016005), and The guidance plan of Natural Science Foundation of Liaoning Province of China (20180550582). None of the funders had any influence on the study design; in the collection, analysis, and interpretation of data; in the writing of the report; and in the decision to submit the article for publication.

\section{ACKNOWLEDGMENTS}

We thank Prof. Leandro Fórnias Machado de Rezende from Department of Preventive Medicine, Faculty of Medicine FMUSP, University of São Paulo for assistance in statistical analysis.

\section{SUPPLEMENTARY MATERIAL}

The Supplementary Material for this article can be found online at: https://www.frontiersin.org/articles/10.3389/fendo. 2019.00617/full\#supplementary-material

12. Shea BJ, Reeves BC, Wells G, Thuku M, Hamel C, Moher D, et al. AMSTAR 2: a critical appraisal tool for systematic reviews that include randomised or non-randomised studies of healthcare interventions, or both. BMJ. (2017) 358:j4008. doi: 10.1136/bmj.j4008

13. Higgins JP, Thompson SG, Deeks JJ, Altman DG. Measuring inconsistency in meta-analyses. BMJ. (2003) 327:557-60. doi: 10.1136/bmj.327.7414.557

14. Ioannidis JP, Patsopoulos NA, Evangelou E. Uncertainty in heterogeneity estimates in meta-analyses. BMJ. (2007) 335:914-16. doi: 10.1136/bmj.39343.408449.80

15. Riley RD, Higgins JP, Deeks JJ. Interpretation of random effects meta-analyses. BMJ. (2011) 342:d549. doi: 10.1136/bmj.d549

16. Sterne JA, Sutton AJ, Ioannidis JP, Terrin N, Jones DR, Lau J, et al. Recommendations for examining and interpreting funnel plot asymmetry in meta-analyses of randomised controlled trials. BMJ. (2011) 343:d4002. doi: 10.1136/bmj.d4002

17. Ioannidis JP, Trikalinos TA. An exploratory test for an excess of significant findings. Clin Trials. (2007) 4:245-53. doi: 10.1177/1740774507079441

18. Salanti G, Ioannidis JP. Synthesis of observational studies should consider credibility ceilings. J Clin Epidemiol. (2009) 62:115-22. doi: 10.1016/j.jclinepi.2008.05.014

19. Kyrgiou M, Kalliala I, Markozannes G, Gunter MJ, Paraskevaidise E, Gabra H, et al. Adiposity and cancer at major anatomical sites: umbrella review of the literature. BMJ. (2017) 356:j477. doi: 10.1136/bmj.j477

20. Markozannes G, Tzoulaki I, Karli D, Evangelou E, Ntzani E, Gunter MJ, et al. Diet, body size, physical activity and risk of prostate cancer: an umbrella review of the evidence. Eur J Cancer. (2016) 69:61-9. doi: 10.1016/j.ejca.2016.09.026

21. Franciosi M, Lucisano G, Lapice E, Strippoli GF, Pellegrini F, Nicolucci A. Metformin therapy and risk of cancer in patients with type 2 diabetes: systematic review. PloS ONE. (2013) 8:e71583. doi: 10.1371/journal.pone.0071583

22. Lega IC, Shah PS, Margel D, Beyene J, Rochon PA, Lipscombe LL. The effect of metformin on mortality following cancer among patients with diabetes. Cancer Epidemiol Biomark Prev. (2014) 23:1974-84. doi: 10.1158/1055-9965.EPI-14-0327

23. Hu J, Chen JB, Cui Y, Zhu YW, Ren WB, Zhou X, et al. Association of metformin intake with bladder cancer risk and oncologic outcomes in type 2 diabetes mellitus patients: a systematic review and meta-analysis. Medicine. (2018) 97:e11596. doi: 10.1097/MD.0000000000011596 
24. Tang GH, Satkunam M, Pond GR, Steinberg GR, Blandino G, Schünemann $\mathrm{HJ}$, et al. Association of metformin with breast cancer incidence and mortality in patients with type II diabetes: a GRADE-assessed systematic review and meta-analysis. Cancer Epidemiol Biomark Prev. (2018) 27:627-35. doi: 10.1158/1055-9965.EPI-17-0936

25. Xu H, Chen K, Jia X, Tian Y, Dai Y, Li D, et al. Metformin use is associated with better survival of breast cancer patients with diabetes: a meta-analysis. Oncologist. (2015) 20:1236-44. doi: 10.1634/theoncologist.2015-0096

26. Mansourian M, Karimi R, Vaseghi G. Different effects of metformin and insulin on primary and secondary chemoprevention of colorectal adenoma in diabetes type 2: traditional and Bayesian meta-analysis. EXCLI J. (2018) 17:45-56. doi: 10.17179/excli2017-993

27. He XK, Su TT, Si JM, Sun LM. Metformin is associated with slightly reduced risk of colorectal cancer and moderate survival benefits in diabetes mellitus: a meta-analysis. Medicine. (2016) 95:e2749. doi: 10.1097/MD.0000000000002749

28. Du L, Wang M, Kang Y, Li B, Guo M, Cheng Z, et al. Prognostic role of metformin intake in diabetic patients with colorectal cancer: an updated qualitative evidence of cohort studies. Oncotarget. (2017) 8:26448-59. doi: 10.18632/oncotarget.14688

29. Zhou XL, Xue WH, Ding XF, Li LF, Dou MM, Zhang WJ, et al. Association between metformin and the risk of gastric cancer in patients with type 2 diabetes mellitus: a meta-analysis of cohort studies. Oncotarget. (2017) 8:55622-31. doi: 10.18632/oncotarget.16973

30. Chu D, Wu J, Wang K, Zhao M, Wang C, Li L, et al. Effect of metformin use on the risk and prognosis of endometrial cancer: a systematic review and meta-analysis. BMC Cancer. (2018) 18:438. doi: 10.1186/s12885-018-4334-5

31. Guo J, Xu K, An M, Zhao Y. Metformin and endometrial cancer survival: a quantitative synthesis of observational studies. Oncotarget. (2017) 8:66169-77. doi: 10.18632/oncotarget.19830

32. Li L, Qi X, Xu M, Ding X, Zhou X, Zhang C, et al. The effects of metformin on ovarian cancer: an updated systematic review and meta-analysis. Int J Clin Exp Med. (2016) 9:17559-68.

33. Li Y, Hu L, Xia Q, Yuan Y, Mi Y. The impact of metformin use on survival in kidney cancer patients with diabetes: a meta-analysis. Int Urol Nephrol. (2017) 49:975-81. doi: 10.1007/s11255-017-1548-4

34. Ma S, Zheng Y, Xiao Y, Zhou P, Tan H. Meta-analysis of studies using metformin as a reducer for liver cancer risk in diabetic patients. Medicine. (2017) 96:e6888. doi: 10.1097/MD.0000000000006888

35. Ma SJ, Zheng YX, Zhou PC, Xiao YN, Tan HZ. Metformin use improves survival of diabetic liver cancer patients: systematic review and meta-analysis. Oncotarget. (2016) 7:66202-11. doi: 10.18632/oncotarget.11033

36. Nie SP, Chen H, Zhuang MQ, Lu M. Anti-diabetic medications do not influence risk of lung cancer in patients with diabetes mellitus: a systematic review and meta-analysis. Asian Pac J Cancer Prev. (2014) 15:6863-9. doi: 10.7314/APJCP.2014.15.16.6863

37. Xin WX, Fang L, Fang QL, Zheng XW, Ding HY, Huang P. Effect of hypoglycemic agents on survival outcomes of lung cancer patients with diabetes mellitus: a meta-analysis. Medicine. (2018) 97:e0035. doi: 10.1097/MD.0000000000010035

38. Hu H, Fang Y, Zhou X, Gong L, Liu L, Wang W, et al. Relationship of metformin with the risk of pancreatic cancer in patients with type 2 diabetes: a meta-analysis. Biomed Res. (2017) 28:4439-44.

39. Zhou DC, Gong H, Tan CQ, Luo JQ. Prognostic significance of antidiabetic medications in pancreatic cancer: a meta-analysis. Oncotarget. (2017) 8:62349-57. doi: 10.18632/oncotarget.17728

40. Xiao Y, Zheng L, Mei Z, Xu C, Liu C, Chu X, et al. The impact of metformin use on survival in prostate cancer: a systematic review and meta-analysis. Oncotarget. (2017) 8:100449-58. doi: 10.18632/oncotarget.22117

41. Torre LA, Bray F, Siegel RL, Ferlay J, Lortet-Tieulent J, Jemal A. Global cancer statistics, 2012. CA Cancer J Clin. (2015) 65:87-108. doi: 10.3322/caac.21262

42. Ryan DP, Hong TS, Bardeesy N. Pancreatic adenocarcinoma. N Eng J Med. (2014) 371:1039-49. doi: 10.1056/NEJMra1404198

43. Decensi A, Puntoni M, Goodwin P, Cazzaniga M, Gennari A, Bonanni B, et al. Metformin and cancer risk in diabetic patients: a systematic review and meta-analysis. Cancer Prev Res. (2010) 3:1451-61. doi: 10.1158/1940-6207.CAPR-10-0157
44. Noto H, Goto A, Tsujimoto T, Noda M. Cancer risk in diabetic patients treated with metformin: a systematic review and meta-analysis. PloS ONE. (2012) 7:e33411. doi: 10.1371/journal.pone.0033411

45. Karnevi E, Said K, Andersson R, Rosendahl AH. Metformin-mediated growth inhibition involves suppression of the IGF-I receptor signalling pathway in human pancreatic cancer cells. BMC Cancer. (2013) 13:235. doi: 10.1186/1471-2407-13-235

46. Sinnett-Smith J, Kisfalvi K, Kui R, Rozengurt E. Metformin inhibition of mTORC1 activation, DNA synthesis and proliferation in pancreatic cancer cells: dependence on glucose concentration and role of AMPK. Biochem Biophys Res Commun. (2013) 430:352-7. doi: 10.1016/j.bbrc.2012.11.010

47. Brenner H, Kloor M, Pox CP. Colorectal cancer. Lancet. (2014) 383:1490-502. doi: 10.1016/S0140-6736(13)61649-9

48. Fransgaard T, Thygesen LC, Gogenur I. Metformin increases overall survival in patients with diabetes undergoing surgery for colorectal cancer. Ann Surg Oncol. (2016) 23:1569-75. doi: 10.1245/s10434-0155028-8

49. Sehdev A, Shih YC, Vekhter B, Bissonnette MB, Olopade OI, Polite BN. Metformin for primary colorectal cancer prevention in patients with diabetes: a case-control study in a US population. Cancer. (2015) 121:1071-8. doi: 10.1002/cncr.29165

50. Pantziarka P, André N. Editorial: drug repurposing. Front Med. (2019) 6:154 doi: 10.3389/fmed.2019.00154

51. Wu T, Thazhath SS, Bound MJ, Jones KL, Horowitz M, Rayner CK. Mechanism of increase in plasma intact GLP-1 by metformin in type 2 diabetes: stimulation of GLP-1 secretion or reduction in plasma DPP-4 activity? Diabetes Res Clin Pract. (2014) 106:e3-6. doi: 10.1016/j.diabres.2014.08.004

52. Lee H, Koa GP. Effect of metformin on metabolic improvement and gut microbiota. Appl Environ Microbiol. (2014) 80:5935-43. doi: 10.1128/AEM.01357-14

53. Lee MS, Hsu CC, Wahlqvist ML, Tsai HN, Chang YH, Huang YC. Type 2 diabetes increases and metformin reduces total, colorectal, liver and pancreatic cancer incidences in Taiwanese: a representative population prospective cohort study of 800,000 individuals. BMC Cancer. (2011) 11:20. doi: 10.1186/1471-2407-11-20

54. Bailey CJ, Day C. Traditional plant medicines as treatments for diabetes. Diabetes Care. (1989) 12:553-64. doi: 10.2337/diacare.12.8.553

55. Yi G, He Z, Zhou X, Xian L, Yuan T, Jia X, et al. Low concentration of metformin induces a p53-dependent senescence in hepatoma cells via activation of the AMPK pathway. Int J Oncol. (2013) 43:1503-10. doi: $10.3892 /$ ijo.2013.2077

56. Dowling RJ, Zakikhani M, Fantus IG, Pollak M, Sonenberg N. Metformin inhibits mammalian target of rapamycin-dependent translation initiation in breast cancer cells. Cancer Res. (2007) 67:10804-12. doi: 10.1158/0008-5472.CAN-07-2310

57. Frasca F, Pandini G, Sciacca L, Pezzino V, Squatrito S, Belfiore A, et al. The role of insulin receptors and IGF-I receptors in cancer and other diseases. Arch Physiol Biochem. (2008) 114:23-37. doi: 10.1080/13813450801969715

58. Belfiore A, Malaguarnera R, Vella V, Lawrence MC, Sciacca L, Frasca $\mathrm{F}$, et al. Insulin receptor isoforms in physiology and disease: an updated view. Endocr Rev. (2017) 38:379-431. doi: 10.1007/978-3-31927318-1

59. Malaguarnera R, Sacco A, Morcavallo A, Squatrito S, Migliaccio A, Morrione $A$, et al. Metformin inhibits androgen-induced IGF-IR up-regulation in prostate cancer cells by disrupting membrane-initiated androgen signaling. Endocrinology. (2014) 155:1207-21. doi: 10.1210/en.2013-1925

60. Vander Heiden MG, Cantley LC, Thompson CB. Understanding the Warburg effect: the metabolic requirements of cell proliferation. Science. (2009) 324:1029-33. doi: 10.1126/science.1160809

61. Boros LG, D'Agostino DP, Katz HE, Roth JP, Meuillet EJ, Somlyai G. Submolecular regulation of cell transformation by deuterium depleting water exchange reactions in the tricarboxylic acid substrate cycle. Med Hypotheses. (2016) 87:69-74. doi: 10.1016/j.mehy.2015.11.016

62. Petrachi T, Romagnani A, Albini A, Longo C, Argenziano G, Grisendi G, et al. Therapeutic potential of the metabolic modulator phenformin in targeting the stem cell compartment in 
melanoma. Oncotarget. (2017) 8:6914-28. doi: 10.18632/oncotarget. 14321

63. Somlyai G, Collins TQ, Meuillet EJ, Hitendra P, D'Agostino DP, Boros LG. Structural homologies between phenformin, lipitor and gleevec aim the same metabolic oncotarget in leukemia and melanoma. Oncotarget. (2017) 8:50187-92. doi: 10.18632/oncotarget. 16238

64. Elgendy M, Cirò, M, Hosseini A, Weiszmann J, Mazzarella L, Ferrari E, et al. Combination of hypoglycemia and metformin impairs tumor metabolic plasticity and growth by modulating the PP2A-GSK3 $\beta$-MCL1 axis. Cancer Cell. (2019) 35:798-815.e5. doi: 10.1016/j.ccell.2019. 03.007

65. Zhuang Y, Chan DK, Haugrud AB, Miskimins WK. Mechanisms by which low glucose enhances the cytotoxicity of metformin to cancer cells both in vitro and in vivo. PLoS ONE. (2014) 9:e108444. doi: 10.1371/journal.pone. 0108444

Conflict of Interest Statement: The authors declare that the research was conducted in the absence of any commercial or financial relationships that could be construed as a potential conflict of interest.

Copyright (c) 2019 Yu, Zhong, Gao, Shi, Wu, Guo, Wang and Song. This is an open-access article distributed under the terms of the Creative Commons Attribution License (CC BY). The use, distribution or reproduction in other forums is permitted, provided the original author(s) and the copyright owner(s) are credited and that the original publication in this journal is cited, in accordance with accepted academic practice. No use, distribution or reproduction is permitted which does not comply with these terms. 